\title{
Revealing the nanoindentation response of a single cell using a 3D structural finite element model
}

\author{
Wenjian Yang ${ }^{1}$, Damien Lacroix ${ }^{2}$, Lay Poh Tan ${ }^{3}$, Jinju Chen ${ }^{1, a)}$ \\ ${ }^{1}$ School of Engineering, Newcastle University, Stephenson Building, Newcastle upon Tyne NE1 7RU, UK \\ ${ }^{2}$ Department of Mechanical Engineering, The University of Sheffield, Pam Liversidge, Mappin Street, Sheffield S1 3JD, UK \\ ${ }^{3}$ School of Materials Science \& Engineering, Nanyang Technological University, 50 Nanyang Avenue, Singapore 639798, Singapore \\ a) Address all correspondence to this author. e-mail: Jinju.chen@ncl.ac.uk \\ Received: 8 June 2020; accepted: 26 October 2020 ; published online: 7 January 2021
}

Jinju Chen was an editor of this journal during the review and decision stage. For the JMR policy on review and publication of manuscripts authored by editors, please refer to http://www.mrs.org/editor-manuscripts/

\begin{abstract}
Changes in the apparent moduli of cells have been reported to correlate with cell abnormalities and disease. Indentation is commonly used to measure these moduli; however, there is evidence to suggest that the indentation protocol employed affects the measured moduli, which can affect our understanding of how physiological conditions regulate cell mechanics. Most studies treat the cell as a homogeneous material or a simple core-shell structure consisting of cytoplasm and a nucleus: both are far from the real structure of cells. To study indentation protocol-dependent cell mechanics, a finite element model of key intracellular components (cortex layer, cytoplasm, actin stress fibres, microtubules, and nucleus) has instead been developed. Results have shown that the apparent moduli obtained with conical indenters decreased with increasing cone angle; however, this change was less significant for spherical indenters of increasing radii. Furthermore, the interplay between indenter geometry and intracellular components has also been studied, which is useful for understanding structure-mechanicsfunction relationships of cells.
\end{abstract}

\section{Introduction}

Knowledge of the mechanical properties of cells is crucial for understanding various cell behaviours including motility [1], differentiation [2], and proliferation [3]. Changes in cell elasticity have been correlated with cell abnormalities and various human diseases including cancer, malaria, and arthritis. Researchers have also shown that the elastic moduli of epithelial cancer cells in the human bladder are lower than those of normal cells due to reorganisation of the cytoskeleton [4]. Furthermore, red blood cells infected with malaria were found to be stiffer than normal cells, which may cause trapping and destruction of the cells in the reticuloendothelial system [5]. Several techniques have been developed to quantify the mechanical properties of living cells, including micropipette aspiration [6], magnetic twisting cytometry [7], optical tweezers [8], and nanoindentation [9]. Atomic force microscope (AFM) is a viable nanoindentation technique capable of revealing the structure and mechanical properties of cells. When integrated with other techniques, it would be even more powerful [10]. For example, AFM combined with fluorescence imaging has been applied to assess the stress fibre amount and stiffness of mouse fibroblasts [11]. Using AFM together with confocal microscopy, the nuclear stiffness of tumour cells was measured with simultaneous visualisation of the indentation process [12]. When combined with microfabricated well arrays, cells can be mechanically entrapped and automated indentation tests can be performed on a number of cells [13]. This requires use of computational modelling or a modified Hertz model to reliably extract the mechanical properties of cells due to the constraints induced by the wall [14].

To better interpret the nanoindentation experimental results, a number of computational models have been developed. Most assumed that cells are a homogenous material [15, 16] or consist of homogenous cytoplasm and a nucleus [17, 18], while excluding the important structure components like actin stress fibres. When using these simplified computational models, however, it is challenging to account for the microstructure 
heterogeneity induced indentation size effect of cells, particularly when using the sharp pyramid AFM probe. Furthermore, due to differences in protocol which aren't accounted for in these simple models, researchers will often find that their measured elasticities contradict what other labs reported for the same types of cell. This significantly hinders the deep understanding of the biomechanics of cells and affects data sharing in the scientific community. It was often concluded that these large discrepancies in the measured apparent cell moduli was due to different methods of sample preparation or variations in individual cells; however, it was also found that the cell modulus measured using AFM probes with different geometries can be vary significantly $[19,20]$. For example, osteoblasts have been probed by Charras and Horton with both pyramidal and spherical indenters, yielding higher values with a pyramidal tip $(E=14.0 \mathrm{kPa})$ than with a spherical indenter $(E=3.2 \mathrm{kPa})[19,20]$. Carl and Schillers also reported higher values when measuring with a sharp probe as compared to when measurements were taken with a spherical one [21]. Vargas-Pinto et al. demonstrated that the stiff cell cortex could provide an explanation for why the apparent modulus was higher when measuring with sharp tips [22]. Stress fibres underlying the cell membrane could be resolved by nanomechanical maps, demonstrating the significant contributions to higher local stiffness of cells [23].

So far, there is a lack of computational modelling which illustrates how the indenter geometry, size and indentation depth all affect the apparent cell modulus when considering the contributions of intracellular components. Therefore, in this study, we aim to develop a comprehensive computational model which considers the key structural components (i.e. cytoplasm, nucleus, cortex, microtubules and actin stress fibres) to study how AFM probe geometry, size and test protocols affect the measured apparent modulus of cells (Figs. 1, 2).

\section{Results}

\section{Indenter size effect on the apparent cell moduli}

To assess the effect of indenter size selection on the apparent Young's moduli determined by Eq. (1) or (2) depending on the indenter geometries, indentation studies with different indenter sizes were performed (Fig. 3). Indentation studies showed a negative correlation between spherical indenter size and apparent modulus. The apparent modulus decreases slightly from $0.85 \mathrm{kPa}$ to $0.62 \mathrm{kPa}$ when the spherical indenter radius was increased from 1 to $5 \mu \mathrm{m}$ (Fig. 3b). When using a conical indenter, however, the measured modulus dramatically drops from 10.21 to $0.96 \mathrm{kPa}$ while increasing the semiincluded angle of the cone (Fig. 3d). The apparent moduli measured by conical indenters are significantly higher (can
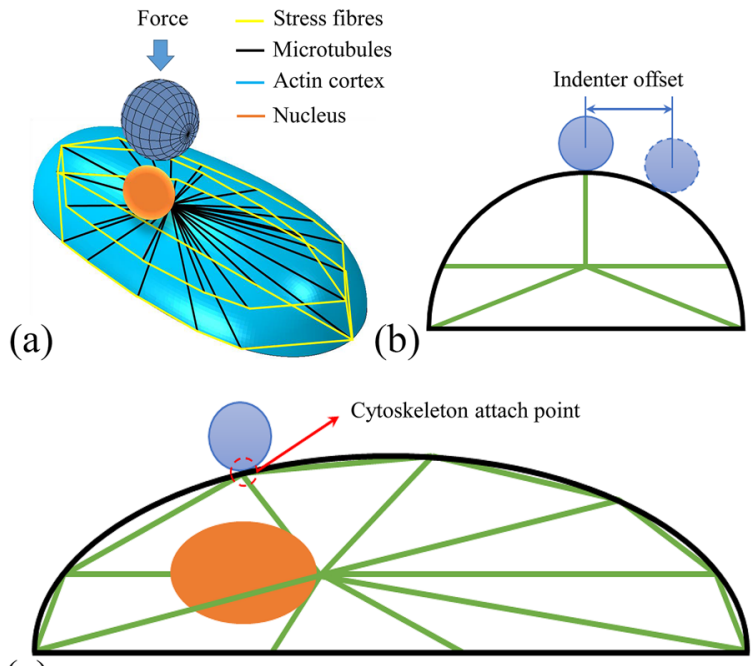

(c)

Figure 1: (a) Cell model consists of stress fibres, microtubules, the actin cortex, cytoplasm, and a nucleus. (b), (c) Schematic views of the indentation positions relative to the cytoskeleton components. The cell is indented around the site where actin stress fibres are attached.

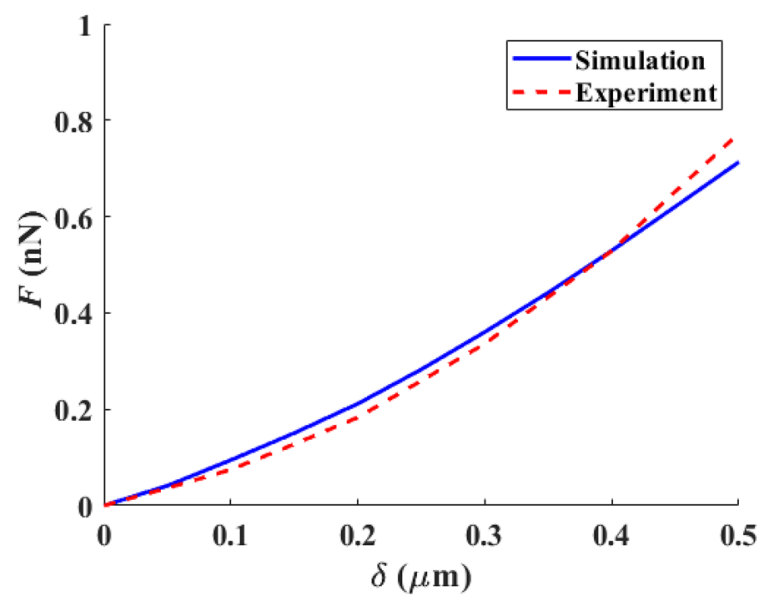

Figure 2: Comparison of the measured force-displacement curve (averaged based on 18 curves [41]) and FE simulation for nanoindentation tests of U2OS cells. There is no significant difference between FE results and the averaged force-displacement curve $(p>0.95)$.

be more than 10 times higher) than the results obtained by their counterpart spherical indenters at given penetrations. The stress map show that, when small, both types of indenter produced higher stresses in the cell cortex, cytoplasm and CSK; large indenters, on the other hand, resulted in greater stress in the nucleus (Figs. S1, S2). 

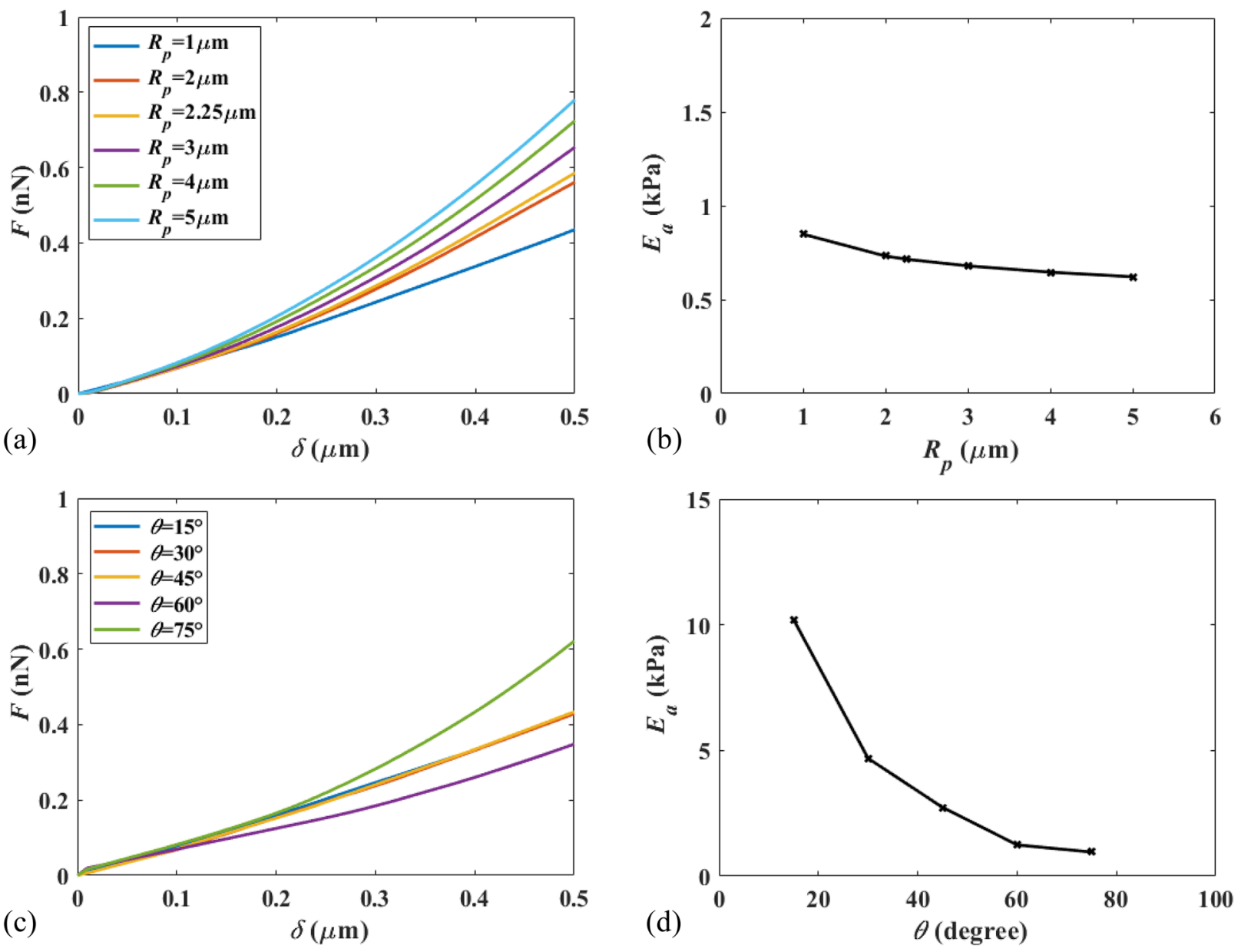

Figure 3: (a), (c) Indentation force $(F)$ versus depth $(\delta)$ of spherical indenters and conical indenters, respectively. (b), (d) Measured apparent Young's moduli $\left(E_{\mathrm{a}}\right)$ versus indenter radius $\left(R_{\mathrm{p}}\right)$ of spherical indenters and semi-included angle $(\theta)$ of conical indenter, respectively.

\section{Indentation depth dependent moduli}

The indentation depth dependent apparent moduli were analysed since the indentation depth also contributes to the apparent moduli (Fig. 4). At a given indenter size, larger indentation depth produced lower and more stable apparent moduli both in spherical and conical indentations. When the radius or semi-included angle of the indenters was increased, the indentation depth dependent apparent moduli tended to converge. At relatively small penetrations, the spherical tip with a smaller radius (e.g. $1 \mu \mathrm{m}$ ) leads to an apparent modulus that is nearly twice that measured with a larger radius indenter (e.g. 2-5 $\mu \mathrm{m}$ ). Modelling also suggested that the apparent moduli measured are very similar at relatively large penetrations (e.g. $0.5 \mu \mathrm{m}$, free from substrate effect) for a spherical probe with radius $3-5 \mu \mathrm{m}$. For the smaller radius probe, the apparent modulus drops by over $60 \%$ when the indentation penetration increases from $0.05 \mu \mathrm{m}$ to $0.5 \mu \mathrm{m}$. On the other hand, the apparent modulus only drops by about $40 \%$ for the probes with larger radius. Such a geometry dependent and indentation size effect is even more pronounced for the conical probes. At shallow penetrations, the sharpest probe leads to the apparent modulus that is nearly 15 times its counterpart measured with a much blunter tip (e.g. $\theta=75^{\circ}$ ). Even at large penetrations (e.g. $0.5 \mu \mathrm{m}$ ), the apparent modulus measured by the sharpest probe is still about 8 times that measured by the bluntest probe in this study.

\section{Indenter position effect on the apparent cell moduli}

To study the cell heterogeneity induced variations in apparent cell moduli, the indenters were displaced from the central position by up to $1 \mu \mathrm{m}$ (Fig. 5). The spherical indentation with $1 \mu \mathrm{m}$ radius appears to be the most sensitive to offset values (the modulus decreases from $0.85 \mathrm{kPa}$ to $0.43 \mathrm{kPa}$ ). In contrast, the apparent moduli measured in studies with other indenter sizes fluctuate in the range of $0.58 \sim 0.73 \mathrm{kPa}$. For sharp conical indenters $\left(\theta=15^{\circ}\right.$ and $\left.30^{\circ}\right)$, the apparent moduli vary greatly from $\sim 9.87$ to $\sim 0.7 \mathrm{kPa}$. The blunt indenters $\left(\theta=60^{\circ}\right.$ and $\left.75^{\circ}\right)$ produced relatively small variations within the range $0.95 \mathrm{kPa}$ to $1.27 \mathrm{kPa}$. The stress maps of intracellular components (Figs. 

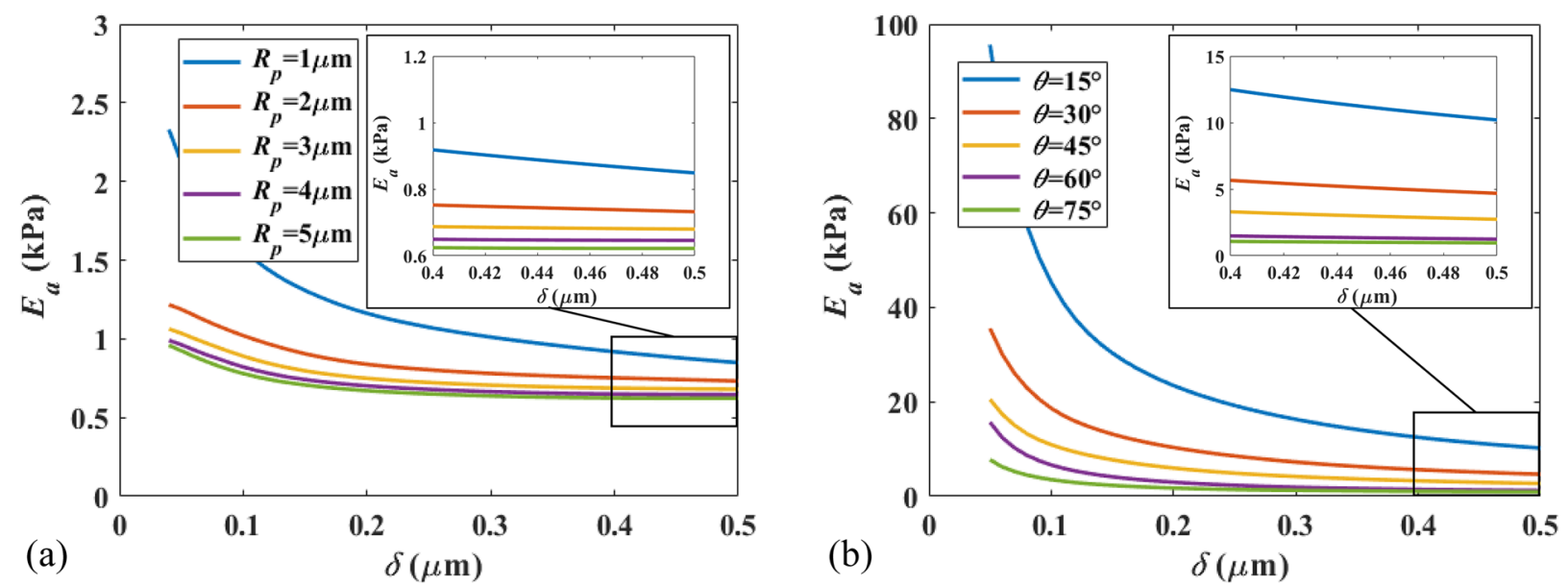

Figure 4: Indentation depth effect on measured apparent moduli with different indenter sizes. (a) Spherical indentation; (b) conical indentation. Insets show the apparent moduli of the cell in the depth $(\delta)$ range of $0.4-0.5 \mu \mathrm{m}$.
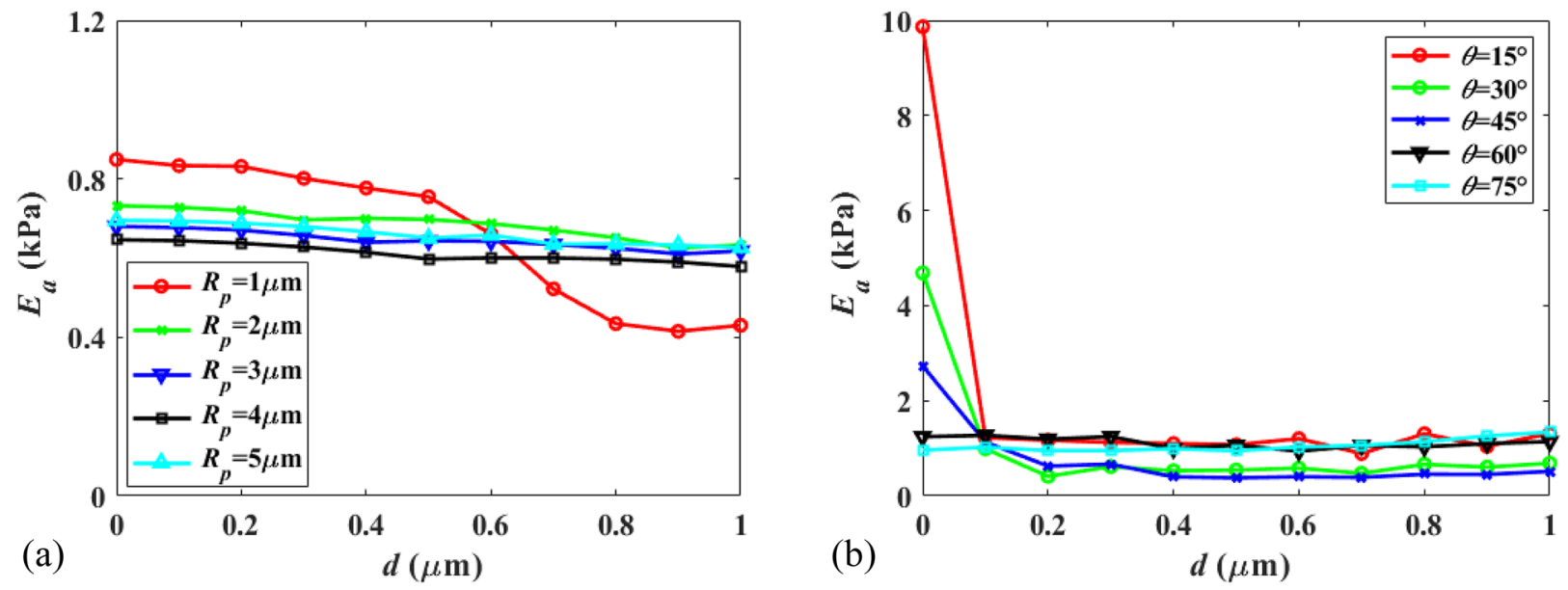

Figure 5: (a) Indentation position effect (with offset $d$ from the stress fibre attach point) on measured apparent Young's moduli with different spherical indenter sizes. The moduli measured by indenters with $R_{\mathrm{p}}>1 \mu \mathrm{m}$ are stable when displacing the tips. For the indenter with $R_{p}=1 \mu \mathrm{m}$, the modulus drops when offset is beyond $0.5 \mu \mathrm{m}$. (b) Indentation position effect (with offset $d$ from the stress fibre attach point) on measured moduli with different conical indenter sizes. For indenters with semi-included angle $\theta<60^{\circ}$, the measured moduli fall dramatically upon displacement.

S1, S2) show much higher stresses when indenting on top of stress fibres.

\section{Heat map of cell modulus}

To link the simulation results with experimental observations, we conducted a mapping analysis by indenting the top surface of the cell model with both types of indenters. Each indentation simulation has been conducted at 64 positions. Heat maps of apparent cell moduli derived by different sizes of indenters are presented in Fig. 6 . The apparent moduli $\left(E_{\mathrm{a}}\right)$ are normalised by the apparent moduli of a control cell model $\left(E_{\mathrm{ctr}}\right)$, in which CSK elements are removed. The noise from FE mesh and model geometry could, therefore, be reduced. Consistent with the results of indenter size and geometry effects in Figs. 3 and
5, the apparent moduli near stress fibres measured by conical probes are significantly higher than those measure using spherical probes. The apparent cell moduli are higher near stress fibres in all cases. As the indenter becomes blunter (e.g. bigger semiincluded angle or larger radius), the effect of stress fibres on localised apparent cell modulus was less significant.

\section{Discussion}

Our results have demonstrated that the apparent modulus decreases with indentation depth. Experimental results have also demonstrated that the apparent moduli decrease with the penetration and reach a plateau at large indentation depth regardless of probe shape and size [24]. It has been suggested that the 


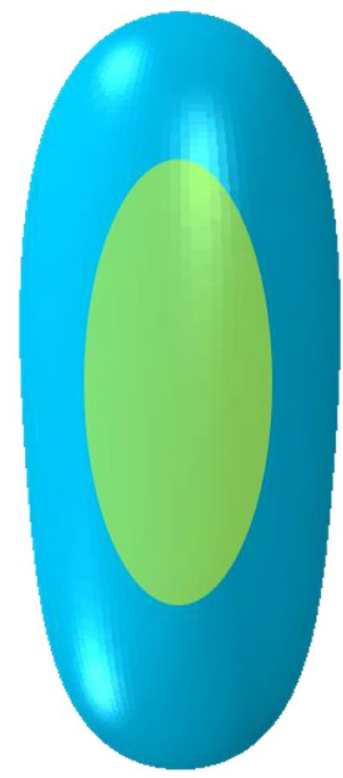

(a)

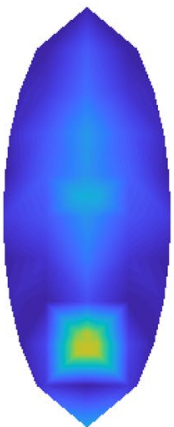

(b) $R_{p}=1 \mu \mathrm{m}$

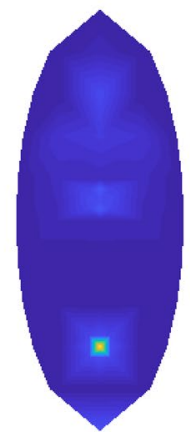

(c) $\theta=15^{\circ}$

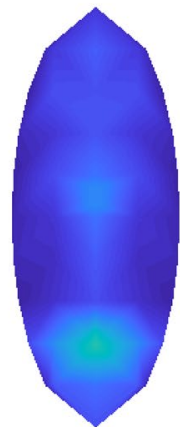

$2 \mu \mathrm{m}$

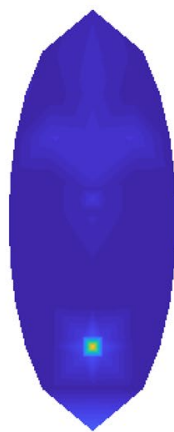

$30^{\circ}$

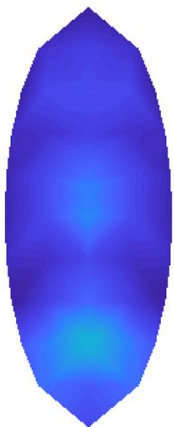

$3 \mu \mathrm{m}$

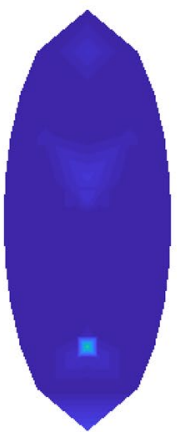

$45^{\circ}$

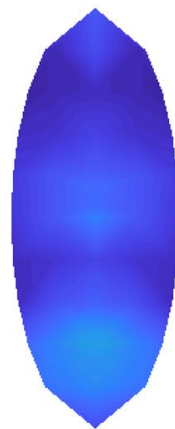

$4 \mu \mathrm{m}$

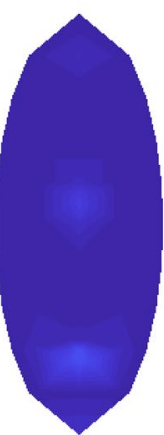

$60^{\circ}$

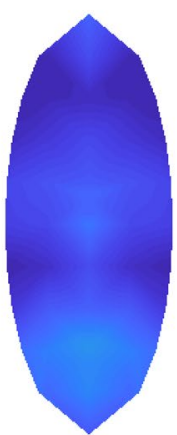

$5 \mu \mathrm{m}$

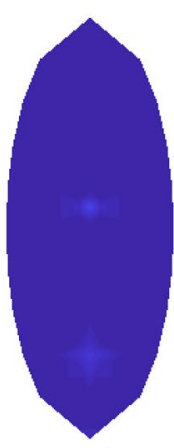

$75^{\circ}$

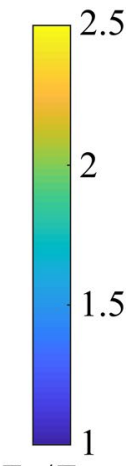

$E_{a} / E_{c t r}$

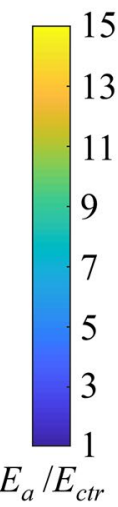

Figure 6: Heat maps of apparent cell moduli measured on the cell surface. (a) The area labelled with green is probed by the indenters. (b) Normalised apparent moduli $\left(E_{\mathrm{a}} / E_{\mathrm{ctr}}\right)$ measured by spherical indenters are averaged by larger sizes. (c) Normalised apparent moduli measured by conical indenters are significantly higher near stress fibres, which are averaged out when using large sizes of probes.

indentation penetration should be restricted to less than $10 \%$ of cell thickness to avoid the effect of the substrate [25]. Within the indentation community, it was often argued that the $10 \%$ rule was generally applicable for hardness measurements [26]. Later, both experimental measurements and numerical simulations demonstrated that this rule is not stringent enough for a hard coating on a very soft substrate and too strict for a soft coating on a hard substrate [27-30]. Thus, such a $10 \%$ rule may not apply to modulus measurements of a thin layer on a substrate because the effective elastic deformation zone is much larger than the plastic deformation zone. Accumulative experimental evidence has, however, demonstrated that the rigid substrate has very little effect on the measured apparent modulus of cells even when the indentation penetration exceeds $10 \%$ or even $25 \%$ of the cell thickness [31]. On the other hand, some other studies reported a stiffening effect with increasing indentation depth [32-34] which was argued to be due to condensation of other cell organelles, e.g. the nucleus and the CSK during indentation. It should be noted that different type of cells can have very different morphologies and internal structure organisations. What was found in one type of cells may not apply to other type of cells.

For the given cell type modelled in this work, it seems that there was no significant effect from the substrate as no stiffening effect was observed at relatively large penetrations (e.g. at maximum penetration, $12 \%$ of the cell thickness). This might not rule out the substrate effect entirely as it could be coupled with the effect of the intracellular components, making it hard to isolate the substrate effect. The apparent softening effect with the indentation penetration was also observed experimentally and it was assumed to be due to stiffer region dominating behaviour at smaller penetrations $[25,35]$. The FE model developed here (Figs. S1, S2) has confirmed such assumption that the indenters initially probe the stiff actin cortex and the CSK, which is significantly stiffer than the cytoplasm. As penetration is increased, the mechanical contribution of the soft cytoplasm also increases.

The cell is generally considered to be heterogeneous due to its different mechanical properties in different cell positions [36]. The heterogeneity of the cell in the model described here was created by attaching the CSK elements onto the cell membrane, enabling the probes to probe the effect of the CSK structure. CSK elements do contribute to the overall cell apparent moduli in our results ( $\sim 0.72 \mathrm{kPa})$ compared to that without CSK $(\sim 0.31 \mathrm{kPa}$, equivalent to the cells with depolymerised actin fibres and microtubules) with spherical indenters (simulation data not shown). When the pyramidal indenters were used, Hofmann et al. also estimated that the region with the highest concentration of stress fibres are 3-10 times stiffer than surrounding areas free of these fibres or with very few fibres [37]. In general, all these quantitatively agree with our 
modelling predictions as shown in Fig. 5b. The commonly used commercial pyramid AFM tips could be likened to the conical tips via their equivalent contact area [38]. The commonly used commercial pyramid AFM probes often have a semi-included angle between $10^{\circ}$ and $70^{\circ}$ and its equivalent conical probe will have slightly higher semi-apical angles based on geometrical calculations. When using these probes in practice, at very small penetration (e.g. $0.05 \mu \mathrm{m}$ ), the sharp pyramid can result in an apparent modulus which is almost 50 times of that generated by a spherical indenter. The commonly used spherical indenters have a radius between 1 and $5 \mu \mathrm{m}$. Even at a relatively large penetration (e.g. $0.5 \mu \mathrm{m}$ ) as adopted in many experimental measurements, the sharp pyramid can still lead to an apparent modulus which doubles that measured by a spherical indenter. These predictions semi-quantitatively agree with experimental measurements [39]. Carl and Schillers attributed the higher apparent modulus measured by sharp tips to the higher applied pressure which results in local strain hardening [21]. Harris and Charras have reported on the underestimation of the contact area of sharp tips due to the potential contact between cell surface and AFM cantilever with indentation depth beyond critical value [39]. This could explain the higher modulus measured by sharp tips with deep indentation. The results provided by Vargas-Pinto et al. showed significantly higher apparent moduli measured using sharp tips with lower penetration $(0.4-0.9 \mu \mathrm{m})$ and longer tips [22]. The higher apparent modulus should, therefore, be attributed to other factors within the cell. The finite element results by Vargas-Pinto et al. suggested that the cortex may contribute to the higher values measured using conical tips [22]. These findings also agreed with our FE predictions about higher apparent moduli near stress fibres.

Together with fluorescence imaging, stiffness maps of cells were obtained using a scanning ion conductance microscope. These show the higher apparent moduli measured in the area with higher concentrations of stress fibres [40]. Nanomechanical maps in a recent study clearly showed the higher apparent moduli near actin stress fibres with increasing indentations [23]. Our computed modulus mapping results are consistent with these experimental observations which emphasises the contribution of stiff stress fibres to apparent cell modulus measurement. All these have demonstrated that the heterogenous cell model constructed here is sufficient to capture many important spatial-dependent cell mechanics at various conditions. For sharp indenters, we demonstrate the dramatic variation in measurements near stress fibres. At a given penetration, the contact areas of spherical indenters (or effective deformed volume) are much larger than those of conical indenters, as seen in Figs. S1 and S2. Thus, the effect of the stress fibres on the localised apparent cell modulus is less significant. If one would like to measure the stiffness of a localised area which can reflect the local concentration of actin fibres, sharp probes such as pyramidal and conical indenters should be employed.

\section{Conclusions and future work}

In conclusion, a comprehensive three-dimensional cellular model which includes the key structural components of the cell has been developed to reveal how the AFM probe geometry and indentation size affect the apparent cell modulus measured during nanoindentation tests. This computational model is capable of predicting the spatial-dependent apparent cell modulus when using probes with different geometries, which cannot be achieved by the commonly used homogenous cell models.

In a semi-quantitative way, the model could predict why, in practice, the apparent modulus measured by commonly used pyramid probes is $2-50$ times that measured by the commonly used spherical probes. This modelling also provides experimentalists a guideline on choosing the appropriate probes according to the research needs. This work has also demonstrated that the blunt conical probes (with semi-included angles above $60^{\circ}$ ) enables measurement of the averaged apparent cell modulus in the same way as what can be achieved with a relatively large spherical probe. This study presented the first 3D computational model for predicting the heat map of the whole apparent cell moduli and has suggested that modulus mapping by sharp conical probes can potentially reveal the relative concentration of stress fibres, which can be used to better understand the biophysics of cells. In principle, it is interesting to discuss the rate-dependent behaviour of the cell. It should be noted that the experimental viscoelastic properties for all the intracellular components in the modelling are not available. It will, therefore, be difficult for us to consider the rate-dependent behaviour of these structure components. Furthermore, for the experimental tests that were compared against, the loading rate was so small $(0.05 \mu \mathrm{m} / \mathrm{s})$ that the apparent moduli of cells were similar to their equilibrium moduli. In the FE modelling, the Young's modulus of each intracellular component is the equilibrium modulus, which makes it sensible to compare our FE against experimental measurements reported in Barreto et al. [41].

In the future, this model can be further extended by considering stress fibre density as measured in the lab and detailed attachment mechanisms of the fibres on the cell membrane to better understand how cells sense the mechanical niche and alter their biomechanics. The understanding of the cells with complicated structure in the present work is valuable for fibre-reinforced engineering materials and many biological tissues (e.g. connective tissues) which can be regarded as fibre-reinforced materials with pre-stresses.

\section{Methods}

\section{FE modelling}

An FE model of a representative adherent human bone osteosarcoma epithelial cells (U2OS) was developed in Abaqus 6.14. 
The same cell geometry as described in Barreto et al. [41, 42] was used, which was also used as a representation of experimental measurements. The cell was constructed as a semi-ellipsoid like shape of $19 \mu \mathrm{m}$ length and $4 \mu \mathrm{m}$ width. Although the real cell consists of various components, the model only considered key intracellular components such as the nucleus, the cytoplasm, the cytoskeleton elements, and a layer of actin cortex attached on the cytoplasm (Fig. 1). It is well known that it is the cortex layer underneath the cell membrane rather than the lipid membrane that contributes to cell mechanics. The cortex layer model $(\sim 0.1 \mu \mathrm{m})$ used here does, however, outcompete the contribution of the cell membrane itself [43]. There is no need to create another $10 \mathrm{~nm}$ layer to deliberately represent the cell membrane. The nucleus was placed $0.5 \mu \mathrm{m}$ away from the bottom surface. This simplified architecture of the intracellular structure in the cell model was created based on microscopic image of actin and microtubules distributions in adherent U2OS [41]. The actin stress fibre, a major component of cytoskeleton was modelled with prestrain, which is reported to be $0.24 \pm 0.18$ [44]. The prestress in the stress fibres is calculated to be $82 \mathrm{kPa}$ and implemented in the model by user subroutine (UMAT). These stress fibres and microtubules were attached onto the cell cortex. The model includes 32 stress fibre elements and 33 microtubules elements in total. Although the number of stress fibres is less than the number in real cells, each fibre can be considered a bundle of fibres which should represent the heterogeneity of the cell structure with good computational efficiency. The microtubules were modelled as star-shape structures emanating from a point near the nucleus membrane (where a centrosome would be in a real cell).

The cytoplasm and nucleus were assigned linear hexahedron elements. Shell elements were used to represent the actin cortex. The stress fibres were modelled as truss elements with circular cross sections attached to the cell cortex nodes at both ends. The microtubules were modelled using beam elements with pipe section: these emanated from a node on the nucleus surface and interacted with the cell cortex and stress fibres at their ends. To study the indenter geometry effect on the apparent cell moduli, two types of indenters were employed in this study: spherical and conical indenter. The spherical indenter radius ranged from 1 to $5 \mu \mathrm{m}$ to investigate how the apparent moduli varied with indenter radius. Semi-included angles of conical indenters ranged from $15^{\circ}$ to $75^{\circ}$ : each had a rounded tip of radius $10 \mathrm{~nm}$. The indenters were modelled as rigid bodies as they are much stiffer than intracellular components.

\section{Apparent materials properties in FE model}

There is no universal mechanical model for a living cell. Poroelastic or spring-dashpot based viscoelastic models have proven
TABLE 1: Material properties of cellular components in the FE model.

\begin{tabular}{lccc}
\hline \hline & Poisson's ratio & Young's modulus & Number of elements \\
\hline Cytoplasm & 0.48 & $0.25 \mathrm{kPa}[50]$ & 95,150 \\
Nucleus & 0.3 & $1 \mathrm{kPa}[51]$ & 5484 \\
Actin cortex & 0.3 & $2 \mathrm{kPa}[52]$ & 11,748 \\
Microtubules & 0.3 & $2 \mathrm{GPa}[53]$ & 277 \\
Stress fibres & 0.3 & $340 \mathrm{kPa}[44]$ & 35 \\
\hline \hline
\end{tabular}

useful in many cases but can also fail to work in a few cases [45]. Individual intracellular component would also exhibit timedependent mechanical characteristics; however, these properties are difficult to be measured separately. Instead of assuming the time-dependent mechanical parameters based on viscoelastic or poroelastic models, we would prefer to choose a simple material model with only two materials properties (apparent Young's modulus and Poisson's ratio). Thus, all the cell components are assumed to be homogeneous, isotropic and elastic. Their elastic properties are summarised in Table 1, which were taken from experimental measurements or theoretical estimations reported in literature. In the present study, focus was directed towards the contribution of intracellular components on the apparent cell modulus. This isn't currently well understood.

\section{Boundary conditions}

During experimental measurements, cells are often immobilised on a coverslip surface. The ventral surface of the cell was, therefore, fixed to prevent detachment from the coverslip. The nucleus, encapsulated in cytoplasm, was assumed to be bonded to the cytoplasm. An attachment point was defined in the contact area to allow the cytoskeleton to transfer external forces (Fig. 1c). Indentation of $0.5 \mu \mathrm{m}$ was applied at the top the nucleus (equivalent to what was used in prior experimental set-ups [41]). To quantify how the cell heterogeneity influences the apparent moduli, the indentation position on the cell surface was changed (Fig. 1b). The indenter was subsequently displaced $0.1-1 \mu \mathrm{m}$ from the original position.

\section{Model calibration}

Prior to comprehensive study, the model was validated against experiment data taken from [41]. For experimental measurements, nanoindentation tests of $\mathrm{U} 2 \mathrm{OS}$ cells $(N=18)$ were performed using spherical AFM probe of diameter $4.5 \mu \mathrm{m}$. As individual cells differ from one another, the force-displacement curves spread out. The force-displacement curves based on the 18 measurements reported in [41] were, therefore, averaged so that they could be compared to FE results. The loading rate in the experiments was so small $(0.05 \mu \mathrm{m} / \mathrm{s})$ that the apparent moduli of cells were similar to their equilibrium moduli. In the modelling, the Young's modulus of each intracellular component was set to the equilibrium 
modulus. It is, therefore, sensible to make direct comparison. As seen in Fig. 2, there is no significant difference between the FE results and the averaged force-displacement curve $(p>0.95)$. The reported apparent cell modulus was $1.3 \pm 0.8 \mathrm{kPa}$ based on 18 measurements [41]. The apparent cell modulus determined by FE is approximately $0.7 \mathrm{kPa}$ which agrees with experimental results $(1.3 \pm 0.8 \mathrm{kPa})$ within measurement errors.

\section{Data analysis}

The Hertz model has been widely adopted to interpret the AFM indentation results $[32,46,47]$. The materials evaluated in the Hertz model are assumed to be homogenous. Although the cell model in our study is inhomogeneous and the cell may experience a large deformation at larger penetrations, the Hertz model was still adopted to obtain the apparent cell modulus so that direct comparisons with experimental results reported in literature could be made. For the spherical indenter, the following formula was used [48]:

$$
F=\frac{4}{3} \frac{E_{\mathrm{a}}}{1-v^{2}} \sqrt{R} \delta^{3 / 2},
$$

where $F$ is the reaction force of the indenter, $\delta$ is indentation depth, and $v$ is Poisson's ratio. $E_{\mathrm{a}}$ is the apparent cell modulus as a rigid indenter was used. $R$ is the effective radius calculated from the spherical indenter $\left(R_{\mathrm{p}}\right)$ and curvature of cell $\left(R_{\mathrm{c}}\right)$ by the equation $1 / R=1 / R_{\mathrm{p}}+1 / R_{\mathrm{c}}$.

For the conical indenters, the force-displacement relationship was given based on Sneddon's work [49]:

$$
F=\frac{2}{\pi} \frac{E_{\mathrm{a}} \cdot \tan \theta}{1-v^{2}} \delta^{2}
$$

where $\theta$ denotes the semi-included angle of the conical indenter.

\section{Acknowledgments}

WJ acknowledges the Newcastle-Singapore Collaboration PhD scholarship. DL acknowledges EPSRC grant EP/ K03877X/1. JC acknowledges the EPSRC grant (EP/K039083/1). Jack Dawson is acknowledged for proofreading.

\section{Supplementary information}

The online version of this article (https://doi.org/10.1557/ s43578-020-00004-5) contains supplementary material, which is available to authorised users.

\section{Open access}

This article is licensed under a Creative Commons Attribution 4.0 International License, which permits use, sharing, adaptation, distribution and reproduction in any medium or format, as long as you give appropriate credit to the original author(s) and the source, provide a link to the Creative Commons licence, and indicate if changes were made. The images or other third party material in this article are included in the article's Creative Commons licence, unless indicated otherwise in a credit line to the material. If material is not included in the article's Creative Commons licence and your intended use is not permitted by statutory regulation or exceeds the permitted use, you will need to obtain permission directly from the copyright holder. To view a copy of this licence, visit http://creativecommons.org/ licenses/by/4.0/.

\section{References}

1. A. Diz-Muñoz, D.A. Fletcher, O.D. Weiner, Use the force: membrane tension as an organizer of cell shape and motility. Trends Cell Biol. 23(2), 47-53 (2013)

2. O. Chaudhuri, D.J. Mooney, Stem-cell differentiation: anchoring cell-fate cues. Nat. Mater. 11(7), 568 (2012)

3. M.G. Haugh, C.M. Murphy, R.C. McKiernan, C. Altenbuchner, F.J. O'Brien, Crosslinking and mechanical properties significantly influence cell attachment, proliferation, and migration within collagen glycosaminoglycan scaffolds. Tissue Eng. A 17(9-10), 1201-1208 (2011)

4. M. Lekka, P. Laidler, D. Gil, J. Lekki, Z. Stachura, A.Z. Hrynkiewicz, Elasticity of normal and cancerous human bladder cells studied by scanning force microscopy. Eur. Biophys. J. 28(4), 312-316 (1999)

5. G.B. Nash, E. O’Brien, E.C. Gordon-Smith, J.A. Dormandy, Abnormalities in the mechanical properties of red blood cells caused by Plasmodium falciparum. Blood 74(2), 855-861 (1989)

6. S.J. Tan, Q. Li, C.T. Lim, Manipulation and isolation of single cells and nuclei. Methods Cell Biol. 98, 79-96 (2010)

7. M. Puig-de-Morales-Marinkovic, K.T. Turner, J.P. Butler, J.J. Fredberg, S. Suresh, Viscoelasticity of the human red blood cell. AJP Cell Physiol 293(2), C597-C605 (2007)

8. M. Dao, C.T. Lim, S. Suresh, Mechanics of the human red blood cell deformed by optical tweezers. J. Mech. Phys. Solids 51(11-12), 2259-2280 (2003)

9. Q.S. Li, G.Y.H. Lee, C.N. Ong, C.T. Lim, AFM indentation study of breast cancer cells. Biochem. Biophys. Res. Commun. 374(4), 609-613 (2008)

10. S. Marcotti, G.C. Reilly, D. Lacroix, Effect of cell sample size in atomic force microscopy nanoindentation. J. Mech. Behav. Biomed. Mater. 94, 259-266 (2019)

11. N. Gavara, R.S. Chadwick, Relationship between cell stiffness and stress fiber amount, assessed by simultaneous atomic force microscopy and live-cell fluorescence imaging. Biomech. Model. Mechanobiol. 15(3), 511-523 (2016) 
12. K. Marina, R. Joostte, W. Katarina, Probing the compressibility of tumor cell nuclei by combined atomic force-confocal microscopy. Phys. Biol. 10(6), 065002 (2013)

13. L. Ng, H.-H. Hung, A. Sprunt, S. Chubinskaya, C. Ortiz, A. Grodzinsky, Nanomechanical properties of individual chondrocytes and their developing growth factor-stimulated pericellular matrix. J. Biomech. 40(5), 1011-1023 (2007)

14. J.J. Chen, Understanding the nanoindentation mechanisms of a microsphere for biomedical applications. J. Phys. D 46(49), 495303 (2013)

15. J. Chen, G. Lu, Finite element modelling of nanoindentation based methods for mechanical properties of cells. J. Biomech. 45(16), 2810-2816 (2012)

16. Y.M. Efremov, W.-H. Wang, S.D. Hardy, R.L. Geahlen, A. Raman, Measuring nanoscale viscoelastic parameters of cells directly from AFM force-displacement curves. Sci. Rep. 7(1), 1541 (2017)

17. Y. Liu, K. Mollaeian, J. Ren, Finite element modeling of living cells for AFM indentation-based biomechanical characterization. Micron 116, 108-115 (2019)

18. G. Ofek, R.M. Natoli, K.A. Athanasiou, In situ mechanical properties of the chondrocyte cytoplasm and nucleus. J. Biomech. 42(7), 873-877 (2009)

19. G.T. Charras, M.A. Horton, Determination of cellular strains by combined atomic force microscopy and finite element modeling. Biophys. J. 83(2), 858-879 (2002)

20. G.T. Charras, M.A. Horton, Single cell mechanotransduction and its modulation analyzed by atomic force microscope indentation. Biophys. J. 82(6), 2970-2981 (2002)

21. P. Carl, H. Schillers, Elasticity measurement of living cells with an atomic force microscope: data acquisition and processing. Pflügers Arch. Eur. J. Physiol. 457(2), 551 (2008)

22. R. Vargas-Pinto, H. Gong, A. Vahabikashi, M. Johnson, The effect of the endothelial cell cortex on atomic force microscopy measurements. Biophys. J. 105(2), 300-309 (2013)

23. C.R. Guerrero, P.D. Garcia, R. Garcia, Subsurface imaging of cell organelles by force microscopy. ACS Nano 13(8), 9629-9637 (2019)

24. Y. Ding, J. Wang, G.-K. Xu, G.-F. Wang, Are elastic moduli of biological cells depth dependent or not? Another explanation using a contact mechanics model with surface tension. Soft Matter 14(36), 7534-7541 (2018)

25. A.B. Mathur, A.M. Collinsworth, W.M. Reichert, W.E. Kraus, G.A. Truskey, Endothelial, cardiac muscle and skeletal muscle exhibit different viscous and elastic properties as determined by atomic force microscopy. J. Biomech. 34(12), 1545-1553 (2001)

26. G.N. Peggs, I.C. Leigh, Recommended Procedure for Microindentation Vickers Hardness Test (UK National Physical Laboratory, Teddington, 1983)
27. X. Cai, H. Bangert, Hardness measurements of thin filmsdetermining the critical ratio of depth to thickness using FEM. Thin Solid Films 264(1), 59-71 (1995)

28. J. Chen, S.J. Bull, On the factors affecting the critical indenter penetration for measurement of coating hardness. Vacuum 83(6), 911-920 (2009)

29. N. Panich, Y. Sun, Effect of penetration depth on indentation response of soft coatings on hard substrates: a finite element analysis. Surf. Coat. Technol. 182(2-3), 342-350 (2004)

30. Z.-H. Xu, D. Rowcliffe, Finite element analysis of substrate effects on indentation behaviour of thin films. Thin Solid Films 447, 399-405 (2004)

31. R.E. Mahaffy, C.K. Shih, F.C. MacKintosh, J. Käs, Scanning probe-based frequency-dependent microrheology of polymer gels and biological cells. Phys. Rev. Lett. 85(4), 880 (2000)

32. N. Guz, M. Dokukin, V. Kalaparthi, I. Sokolov, If cell mechanics can be described by elastic modulus: study of different models and probes used in indentation experiments. Biophys. J. 107(3), 564-575 (2014)

33. C. Roduit, S. Sekatski, G. Dietler, S. Catsicas, F. Lafont, S.J. Kasas, Stiffness tomography by atomic force microscopy. Biophys. J. 97(2), 674-677 (2009)

34. H. Schillers, M. Wälte, K. Urbanova, H. Oberleithner, Real-time monitoring of cell elasticity reveals oscillating myosin activity. Biophys. J. 99(11), 3639-3646 (2010)

35. A.A. Soufivand, M. Soleimani, M. Navidbakhsh, Is it appropriate to apply Hertz model to describe cardiac myocytes' mechanical properties by atomic force microscopy nanoindentation? Micro Nano Lett. 9(3), 153-156 (2014)

36. J. Solon, I. Levental, K. Sengupta, P.C. Georges, P.A. Janmey, Fibroblast adaptation and stiffness matching to soft elastic substrates. Biophys. J. 93(12), 4453-4461 (2007)

37. U.G. Hofmann, C. Rotsch, W.J. Parak, M. Radmacher, Investigating the cytoskeleton of chicken cardiocytes with the atomic force microscope. J. Struct. Biol. 119(2), 84-91 (1997)

38. S. Sagadevan, P. Murugasen, Novel analysis on the influence of tip radius and shape of the nanoindenter on the hardness of materials. Procedia Mater. Sci. 6, 1871-1878 (2014)

39. A.R. Harris, G.T. Charras, Experimental validation of atomic force microscopy-based cell elasticity measurements. Nanotechnology 22(34), 345102 (2011)

40. J. Rheinlaender, T.E. Schäffer, Mapping the mechanical stiffness of live cells with the scanning ion conductance microscope. Soft Matter 9(12), 3230-3236 (2013)

41. S. Barreto, C.H. Clausen, C.M. Perrault, D.A. Fletcher, D. Lacroix, A multi-structural single cell model of force-induced interactions of cytoskeletal components. Biomaterials 34(26), 6119-6126 (2013)

42. S. Barreto, C.M. Perrault, D. Lacroix, Structural finite element analysis to explain cell mechanics variability. J. Mech. Behav. Biomed. Mater. 38, 219-231 (2014) 
43. G.A. Clark, K. Dierkes, E.K. Paluch, Monitoring actin cortex thickness in live cells. Biophys. J. 105(3), 570-580 (2013)

44. S. Deguchi, T. Ohashi, M.J. Sato, Tensile properties of single stress fibers isolated from cultured vascular smooth muscle cells. J. Biomech. 39(14), 2603-2610 (2006)

45. J. Chen, Nanobiomechanics of living cells: a review. Interface Focus 4(2), 20130055 (2014)

46. Y. Ding, G.-K. Xu, G.-F. Wang, On the determination of elastic moduli of cells by AFM based indentation. Sci. Rep. 7, 45575 (2017)

47. H. Liu, Y. Sun, C.A. Simmons, Determination of local and global elastic moduli of valve interstitial cells cultured on soft substrates. J. Biomech. 46(11), 1967-1971 (2013)

48. K. Johnson, Contact Mechanics (Cambridge University Press, London, 1985)
49. I.N. Sneddon, The relation between load and penetration in the axisymmetric Boussinesq problem for a punch of arbitrary profile. Int. J. Eng. Sci. 3(1), 47-57 (1965)

50. D. Ingber, Opposing Views on Tensegrity as a Structural Framework for Understanding Cell Mechanics-Rebuttals (American Physiological Society, Bethesda, 2000)

51. F. Guilak, V.C.J. Mow, The mechanical environment of the chondrocyte: a biphasic finite element model of cell-matrix interactions in articular cartilage. J. Biomech. 33(12), 1663-1673 (2000)

52. J. Stricker, T. Falzone, M.L.J. Gardel, Mechanics of the F-actin cytoskeleton. J. Biomech. 43(1), 9-14 (2010)

53. F. Pampaloni, E. Florin, Microtubule architecture: inspiration for novel carbon nanotube-based biomimetic materials. Trends Biotechnol. 26(6), 302-310 (2008) 